\title{
ROSSBY NUMBER OR ROTATION PERIOD?
}

\author{
K.STEPIEŃ \\ Astrophysical Institute Potsdam, An der Sternwarte 16 \\ D-O-1561 Potsdam \\ and \\ Warsaw University Observatory, Al. Ujazdowskie 4 \\ 00-478 Warszawa
}

\begin{abstract}
It is shown that the scaling of rotation periods by a color-dependent parameter (turnover time) improves substantially the observed activity- period relations only for single, main sequence, solar type stars with $0.5 \leqslant B-V \leqslant 0.8$. For other single main sequence stars and for single giants activity indices correlate equally well with rotation period and the Rossby number, or show no correlation with either parameter.
\end{abstract}

Key words: stellar activity - stellar rotation

\section{Introduction}

The close relationship between chromospheric activity and rotation of stars (Kraft 1967) has usually been explained as resulting from the rotation dependent dynamo action generating magnetic fields which then influence the activity (e.g. Hartmann and Noyes 1987). According to early, linear dynamo theories the efficiency of dynamo should scale as $\tau_{c} \Omega$, where $\tau_{c}$ is the turnover time in those stellar convective layers where fields are generated, and $\Omega$ is angular velocity of the star (Durney and Latour 1978). The discovery by Noyes et al. (1984) that the observed calcium emission flux scales as the so called Rossby number, $R o=P_{\text {rot }} / \tau_{c}$, where $P_{\text {rot }}$ is the surface rotation period and $\tau_{c}$ refers to the bottom layers of the convection zone was treated as a confirmation of these theoretical predictions. The argument of Noyes et al. (1984) for using the Rossby number as a correct rotation measure was empirical: the plot of the calcium emission flux scaled by the bolometric flux, versus $\log P_{\text {rot }}$ produced a large, color-dependent scatter. When $P_{\text {rot }}$ was replaced by $R o$ the systematic, color-dependent shifts disappeared and the scatter decreased. Since that paper, Ro has become a standard parameter, with which different activity measures are being correlated.

However, not all observers accepted $R o$ as a better measure of rotation than $P_{\text {rot }}$, arguing that the apparent scatter depends both on the choice of abscissa and ordinate, hence a properly selected ordinate can also decrease substantially the scatter (Basri 1986, Rutten 1987, Young et al. 1989, Schrijver 1992). In addition, the recent results on nonlinear theory of rotating convective envelopes predict that, for the observed rates of stellar rotation, $\alpha$ - effect as well as the amount of differential rotation should depend only weakly on the Rossby number (Kichatinov and Ruediger 1992, Ruediger and Elstner 1992). It seems therefore reasonable to rediscuss the observational arguments for using $R o$ as a correct measure of rotation in respect to activity.

In the following, only single stars will be discussed (dwarfs and giants separately), and it will be assumed that differences in activity among stars of the same 
spectral class and the same $B-V$ result solely from different rotation rate. The data are discussed in diagrams where logarithm of activity index is plotted versus $P_{\text {rot }}\left(\right.$ not $\left.\log P_{\text {rot }}\right)$. Linear fits on such diagrams correspond to exponential relations: $\log ($ act. index $)=a-b P_{\text {rot }}$, and the title question of the present paper reduces to the problem whether activity-rotation relations for different $B-V$ are parallel or inclined to one another. In the second case a proper scaling of $P_{\text {rot }}$ by color-dependent factors will make them parallel, introducing thus defined $R_{0}$ in place of $P_{\text {rot }}$. Any residual vertical shifts can be removed by a proper selection of ordinate.

\section{Activity-rotation relations}

\subsection{Main Sequence Stars}

Observations show that the chromospheric-coronal activity can be detected even in dwarfs with $B-V \approx 0.3$ but the activity level of the hottest active stars, i.e. stars with $B-V \lesssim 0.4$ varies very little from one star to another and does not show any correlation with rotation (Schmitt et al. 1985, Wolff et al. 1986, Rutten 1986, Simon and Landsman 1987, Garcia Lopez et al. 1991). For cooler stars, with $0.4 \lesssim B-V$ $\lesssim 0.5$, activity decreases with rotation rate but a large, random scatter appears, which cannot be reduced by any scaling of $P_{\text {rot }}$ discussed here.

Still cooler stars, with $B-V \gtrsim 0.5$, show tight activity-rotation relations with a color-dependent inclination. Fig. 1 (left) demonstrates this for the Noyes et al. (1984) data, divided into 7 spectral intervals with average $B-V$ indicated. Defining $R o \equiv b R_{\text {rot }}$, where $b$ is the empirically determined inclination of a corresponding linear fit in Fig. 1 (left), and using it as an abscissa removes most of the scatter, as can be seen in Fig. 1 (right). Note that in this case the coefficient $a$ of the linear fit is nearly color independent thus the residual scatter depending on the choice of ordinate is small (for a more detailed discussion see Stȩpien 1989).

Fig. 1 shows, however, that $b$ varies with color only for stars with $B-V \leqslant 0.8$ and remains essentially constant for cooler stars. It means that when only the coolest dwarfs are considered, $R o$ is an equally good rotation measure as $P_{\text {rot }}$. Using differently defined $R o$ for these stars with $\tau_{c}$ determined theoretically and strongly variable with color, as some of works suggest (e.g. Rucinski and VandenBerg 1986) would increase the scatter for these stars..

Other activity indices (e.g. X-ray flux, Mg II h and $\mathrm{k}$ line emission flux or intensity of the surface magnetic field) show similar behavior (Stępien , in preparation). The results lead to the conclusion that using $R o$ as an abscissa reduces the observed scatter in activity levels only for solar type stars, i.e. dwarfs with $0.5 \lesssim B-V \lesssim 0.8$.

\subsection{Giants}

An apparent success in correlating activity of some of main sequence stars with $R o$ prompted many authors to use the same parameter for giants. The turnover times for evolved stars were computed by Gilliland (1985). They are generally longer than for main sequence stars of the same color.

Is the use of the argument per analogiam justified in this case? There exist qualitative theoretical arguments that activity of giants cannot be parametrised in 

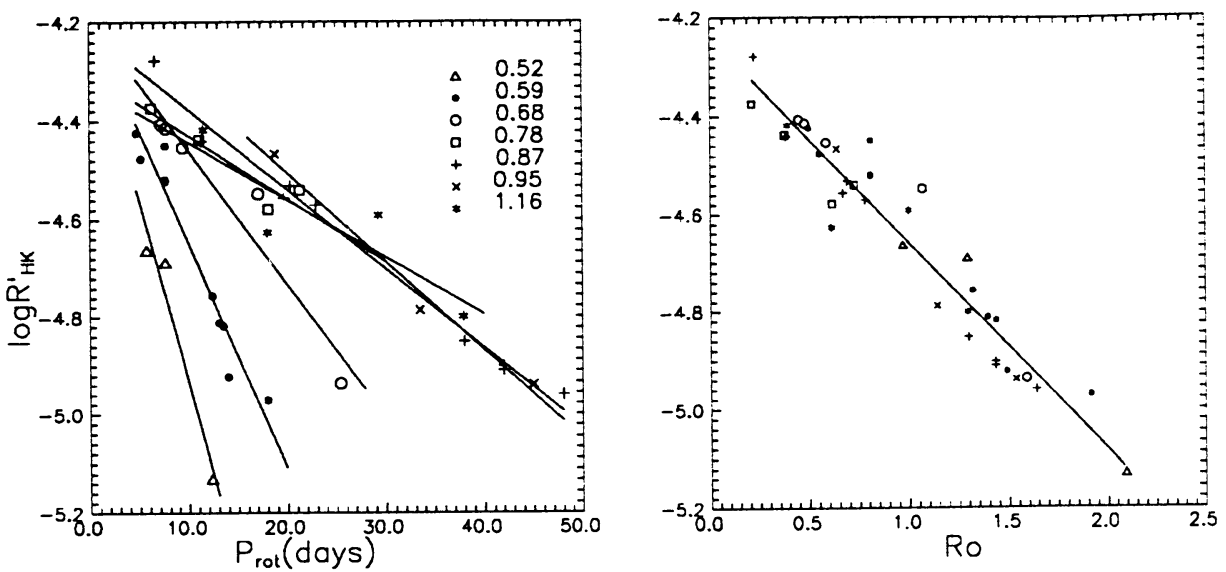

Fig. 1. Original Noyes et al. (1984) data on calcium emission of dwarfs, grouped into $B-V$ intervals with average $B-V$ indicated (three stars with no matching counterparts are omitted), are plotted versus rotation period (left). The right panel shows the same data plotted versus the Rossby number defined in the text.

such a simple way as in case of the main sequence stars. The latter ones have an evolutionary time scale of the order of at least $10^{10}$ years. A time scale for loss of angular momentum during the main sequence life (after a possible rapid spin-down connected with landing on the zero-age-main-sequence) is equal initially $2-3 \times 10^{8}$ years and it lengthens quickly, exceeding $10^{10}$ years by the solar age (Stȩpien 1988). A time scale for redistribution of the internal angular momentum in radiative layers is difficult to determine but several direct and indirect estimates give $10^{8}$ years (Pinsonneault et al. 1989). It may be longer in central parts of the star but this should not influence the dynamo acting in the convection zone, with some possible role played by the radiative layers lying immediately beneath it. The third time scale is thus the shortest of the three and for the nearly whole main sequence life it is much shorter than the other two.A star spinning down evolves hence essentially through equilibrium states of the internal angular momentum distribution. These states can be parametrised e.g. by a surface rotation rate. On the other hand, all structural properties of a main sequence star relevant to dynamo action, like length scales involved, or velocities, can apparently be parametrised by a single parameter, like stellar mass, $T_{e}$, or $B-V$.

The evolutionary time scale of giants is much shorter - it is now the shortest of all three involved scales, particularly in phases when a convection zone develops. As a result, the matter with specific angular momentum still depending on initial conditions is rapidly mixed into the expanding convection zone. It seems thus unlikely that the efficiency of dynamo acting in a giant can be parametrised by its spectral type and the observed rotation rate only. It should also depend on the previous evolutionary history of the stellar angular momentum. The observations of activity support this view, although, due to very scanty data on single giants 


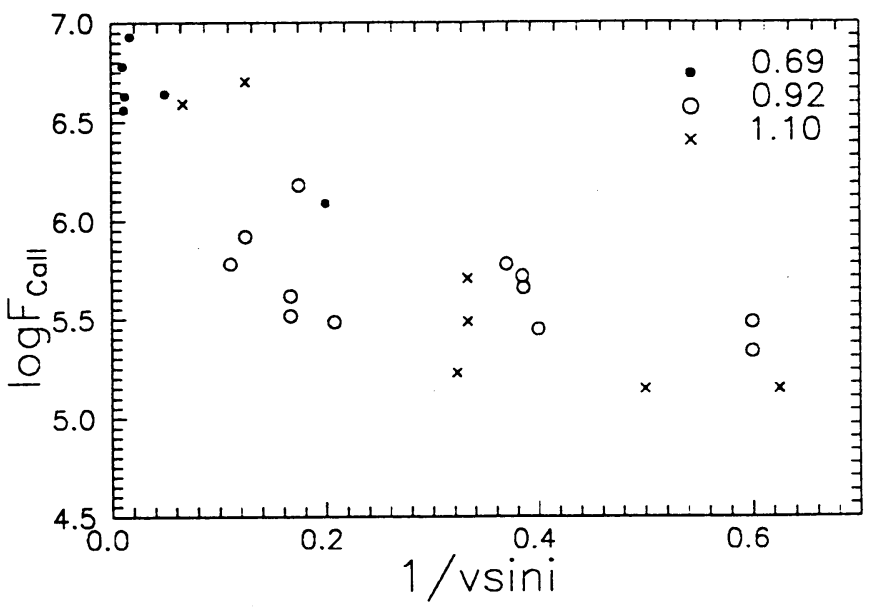

Fig. 2. The surface flux of calcium emission scaled by the bolometric flux, plotted versus $1 / v \sin i \sim P_{\text {rot }}$ for single giants of the indicated colors. The data suggest a fundamentally different rotation dependence of activity than observed for dwarfs.

and a very poor knowledge of their rotation periods, it is not yet possible to draw definite conclusions.

The data on the hottest active giants, with $B-V \leqslant 0.7$, indicate again a narrow range of activity, without any apparent rotation dependence (Simon and Drake 1989, Maggio et al. 1990). Intermediate-color giants, with $0.7 \leqslant B-V \leqslant 1.0-1.1$, show a very broad range of activity, which resembles the solar type dwarfs. However, the giants of one color do not show a linear dependence of $\log$ (act.index) on $P_{\text {rot }}$. Fig. 2 gives an example of such data where the chromospheric calcium emission flux is plotted versus $1 / v \sin i \sim P_{\text {rot }}$. The data suggest that all stars with $1 / v \sin i \leqslant 0.15$ - 0.20 (corresponding to $P_{\text {rot }} \sim 50-100$ days) have a very high level of activity with little or no variation on the rotation rate. At $1 / v \sin i \approx 0.15-0.20$ the activity level rapidly drops and remains nearly constant for slower rotation. This picture must remain only tentative until more accurate data on single giants are available. It agrees, nevertheless, with conclusions of Simon and Drake (1989), and Maggio et al. (1990) who discussed other activity indices and considered a much broader material, with double stars included. Note, that nearly all known RS CVn type stars have rotation periods less than $\sim 100$ days which puts them into a state of high activity, according to the above picture. All known giants with $B-V \gtrsim 1.1$ show a very low level of activity. It is clear that any color-dependent scaling of rotation periods would be irrelevant if the above picture is correct.

\section{Conclusions}

The available observational data on activity suggest that for dwarfs cooler than $B-V \approx 0.5$ activity level decreases exponentially with rotation period. The rate of decrease is a function of color for stars with $B-V \leqslant 0.8$. Using properly defined $R o$ one gets for them a single activity-rotation relation. For cooler dwarfs the rate 
of decrease of activity with rotation rate is color-independent (or, at most, weakly color-dependent), whereas for hotter dwarfs activity level is not correlated with rotation. Giants behave differently: all stars, independently of color, rotating faster than some limit, show a high level of activity, whereas all stars beyond that limit are inactive. The precise location and breadth of this transition requires new, better data. Nevertheless, the Rossby number seems to be irrelevant to activity of giants.

\section{Acknowledgements}

The author would like to thank the Director and the staff of the Astrophysical Institute Potsdam for their hospitality during his stay. This research was partly supported by the grant KBN 211779101.

\section{References}

Basri, G.: 1986, in Cool Stars, Stellar Systems and the Sun, ed(s). M.Zeilik and D.M.Gibson, Springer:Berlin, p. 184

Durney, B.R. and Latour, J.: 1978, Geophys., Astrophys. Fluid Dyn. 9, 241

García Lopéz, .J., Rebolo, R., Beckman, J.E. and Mc Keith, C.D.: 1991, in The Sun and Cool Stars: activity, magnetism, dynamos, ed(s). I.Tuominen et al. , Springer:Berlin, p. 477

Gilliland R.L.: 1985, Ap.J. 299, 286

Hartmann, L.W. and Noyes, R.W.: 1987, Ann.Rev.Astr.Ap. 25, 271

Kichatinov, L.L. and Ruediger, G.: 1992, Astr.Ap. , in print

Kraft, R.P.: 1967, Ap.J. 150, 551

Maggio, A., Vaiana, G.S., Haisch, B.B., Stern, R.A., Bookbinder, J., Harnden, F.R.Jr. and Rosner, R.: 1990, Ap.J. 348, 253

Noyes, R.W., Hartmann, L.W., Baliunas, S.L., Duncan, D.K. and Vaughan, A.H.: 1984, Ap.J. 279, 763

Pinsonneault, M.H., Kawaler, S.D., Sofia, S. and Demarque, P.: 1989, Ap.J. 338, 424

Rucinski, S.M. and VandenBerg, D.A.: 1986, Pub.A.S.P. 98, 669

Ruediger, G, and Elstner, D.: 1992, Astr. Ap. , in print

Rutten, R.G.M.: 1986, Astr.Ap. 159, 291

Rutten, R.G.M.: 1987, Astr.Ap. 177, 131

Schmitt, J.H.M.M., Golub, L., Harnden, F.H.Jr., Maxon, G.W. and Vaiana S.G.: 1985, Ap.J. 290, 307

Schrijver, C.J.: 1992, Proc. of IAU Coll. 137 , in print

Simon, T. and Drake, S.A.: 1989, Ap.J. 346, 303

Simon, T. and Landsman, W.: 1987, in Cool Stars, Stellar Systems and the Sun, ed(s). J.L. Linsky and R.E. Stencel, Springer:Berlin, p. 265

Stępień , K.: 1988, A p.J. 335, 907

Stępień , K.: 1989, Astr.Ap. 210, 273

Wolf, S.C., Boesgaard, A.M. and Simon, T.: 1986, Ap.J. 310, 360

Young, A., Ajir, F. and Thurman, G.: 1989, Pub.A.S.P. 101, 1017 